\title{
ORIGINAL
}

\section{Profiles of atherosclerotic risk factors in gastrectomized men}

\author{
Jun Murai ${ }^{1)}$, Masafumi Koga ${ }^{1)}$, Hiroshi Saito ${ }^{1)}$, Mikio Mukai ${ }^{1)}$, Soeko Matsumoto ${ }^{2)}$ and Soji Kasayama ${ }^{3)}$ \\ 1) Department of Internal Medicine, Kinki Central Hospital, Itami, Japan \\ 2) Department of Laboratory Medicine, Kinki Central Hospital, Itami, Japan \\ ${ }^{3)}$ Department of Medicine, Nissay Hospital, Osaka, Japan
}

\begin{abstract}
Postprandial hyperglycemia is an established risk factor for atherosclerotic vascular diseases, and it is frequently observed in gastrectomized subjects. This study sought to examine whether other atherosclerotic risk factors are also common among gastrectomized subjects. The study population comprised of 44 non-diabetic men who previously underwent gastrectomy. The age- and body mass index-matched control population comprised of 278 non-diabetic men without gastrectomy. In addition to traditional atherosclerotic risk factors for atherosclerosis, high sensitivity C-reactive protein (hsCRP) and brachial-ankle pulse wave velocity (baPWV) were also compared between the groups. Fasting plasma glucose was not different between both groups, while glycated hemoglobin (HbAlc) was significantly higher in the gastrectomized men than in the control men. Systolic and diastolic blood pressures and high density lipoprotein cholesterol (HDL-C) were significantly higher, whereas low density lipoprotein cholesterol (LDL-C) was lower in the gastrectomized men than in the control men. baPWV, hsCRP, triglycerides and insulin resistance (as per the homeostasis model assessment) were not different between groups. While levels of certain atherosclerotic risk factors, including HbA1c and blood pressure are higher among gastrectomized men, HDL-C and LDL-C were actually favorable. Additionally, levels of more emerging risk factors, such as hsCRP and baPWV were not altered among gastrectomized men.
\end{abstract}

Key words: Atherosclerosis, Gastrectomy, Postprandial hyperglycemia, Pulse wave velocity

ORAL glucose tolerance tests (OGTT) among gastrectomized subjects often demonstrate marked hyperglycemia (oxyhyperglycemia) 30 to 60 min after glucose loading $[1,2]$. It has been shown that glycated hemoglobin (HbA1c) is increased and serum glycated albumin (GA) more reflecting postprandial and maximal blood glucose levels is furthermore increased in gastrectomized subjects [3].

Postprandial hyperglycemia or hyperglycemia after oral glucose-loading is a known risk for cardiovascular diseases $[4,5]$. Thus, gastrectomized subjects may be prone to developing atherosclerotic vascular diseases. However, epidemiological studies have reported that these subjects may actually have lower incidence of cardiovascular events [6, 7]. There have been several reports studying the effects of gastrectomy on the tradi-

Received Dec. 20, 2010; Accepted May 16, 2011 as K10E-401

Released online in J-STAGE as advance publication Jun. 10, 2011

Correspondence to: Masafumi Koga, M.D., Ph.D., Department of Internal Medicine, Kinki Central Hospital, Kuruma-zuka 3-1, Itami, Hyogo 664-8533, Japan.

E-mail: koga_m@kich.itami.hyogo.jp tional atherosclerotic risk factors, including blood pressure, serum lipids, insulin resistance, serum uric acids and high sensitivity C-reactive protein (hsCRP) [8-10]. However, it remains unclear whether atherosclerosis preferentially develops in gastrectomized subjects.

Among various surrogate markers for atherosclerosis, pulse wave velocity (PWV) has been shown to have clinical utility as a noninvasive method for predicting future cardiovascular events [11, 12]. In the present study, we sought to investigate whether or not atherosclerotic risk factors including brachial-ankle PWV (baPWV) are commonly present among gastrectomized subjects.

\section{Subjects and Methods}

\section{Study subjects}

During health examinations at Kinki Central Hospital between August 2006 and March 2009, men with a history of gastrectomy were identified. From this group, 44 men whose fasting plasma glucose (FPG) of $<126 \mathrm{mg} / \mathrm{dL}$ were selected as the study group. 
Table 1 Baseline characteristics of participants.

\begin{tabular}{lccc}
\hline & Gastrectomized men & Control men & $p$ value \\
\hline $\mathrm{n}$ & 44 & 278 & - \\
Age (years) & $58.8 \pm 6.8$ & $58.8 \pm 4.3$ & 0.964 \\
BMI (kg/m²) & $22.1 \pm 2.7$ & $22.1 \pm 1.6$ & 0.944 \\
Current smokers (\%) & 25.0 & 26.3 & 0.860 \\
Drinking habits (\%) & 75.0 & 69.8 & 0.482 \\
Duration after gastrectomy (years) & $14.3 \pm 11.3$ & - & - \\
\hline
\end{tabular}

Data presented as mean \pm SD or number. BMI; body mass index.

A total of 1,994 men visited the Health Care Center at Kinki Central Hospital for routine health examination between May 2006 and October 2006. From these subjects, 936 men (mean age; $53.1 \pm 7.4$ years, mean BMI; $23.9 \pm 2.7 \mathrm{~kg} / \mathrm{m}^{2}$ ) without any history of diabetes or evidence of a diabetic pattern on OGTT were identified. In total, 278 age- and BMI-matched men were selected as controls. Exclusion criteria included liver disease (e.g., chronic hepatitis, liver cirrhosis), renal disease, thyroid disease, anemia, administration of glucocorticoid, and treatment for hypertension, dyslipidemia, hyperuricemia and anemia. Clinical characteristics of the study participants are shown in Table 1. Among the 44 gastrectomized men, 18 received gastrectomy because of peptic ulcers while the other 27 had gastric cancers, but were currently confirmed to have no signs of recurrence or metastasis. All study subjects were otherwise healthy with a proper nutritional status. Specifically, subjects had no abnormalities in blood tests, chest X-ray examination and abdominal echography. There were no significant differences in the prevalence of current smokers and that of drinking habits between both groups.

The study was performed in accordance with the principles of the Declaration of Helsinki as revised in 2000, and the study protocol was approved by the ethics committee of Kinki Central Hospital.

\section{Laboratory analyses}

Body height and weight were measured on the same day during the health examination. Blood pressure was measured with an automatic sphygmomanometer with the subject in a sitting position after 5 min of rest. Fasting blood was collected from subjects between 9 $\mathrm{AM}$ and $11 \mathrm{AM}$, and was centrifuged immediately for measurements. Plasma glucose, serum insulin, serum uric acid, total cholesterol, high density lipoprotein cholesterol (HDL-C) cholesterol, and triglycerides were determined by means of standard laboratory assays. HbA1c was measured with ADAMS-A1c HA-8160 (Arkray Inc., Kyoto, Japan) by HPLC, as described previously [13]. HbA1c (\%) is estimated as a National Glycohemoglobin Standardization Program (NGSP) equivalent value (\%), calculated by the formula HbA1c $(\%)=$ HbA1c (Japan Diabetes Society: JDS) (\%) + $0.4 \%$. This formula adjusts for the relative expression of HbA1c (JDS) (\%) to a previous Japanese standard substance for HbA1c (NGSP) [14]. Serum low density lipoprotein cholesterol (LDL-C) levels were calculated using the Friedewald equation [15]. hsCRP was determined by means of latex-enhanced immunonephelometrics on a BNII Analyzer (Dade Behring, Marburg, $\mathrm{GmbH}$ ), as described previously [16]. Insulin resistance (IR) was estimated by the homeostasis model assessment-IR (HOMA-IR) [fasting insulin (pmol/L) x FPG (mmol/L)]/135 [17]. Ankle-brachial blood pressure index (ABI) and baPWV were measured with the use of a Waveform analyzer (VaSera VS-1000, Fukuda Denshi, Tokyo, Japan), after 5 min rest [18]. The validity and reproducibility of the baPWV measurements have been previously reported [19]. In our laboratory, the coefficients of variations of inter-observer and intra-observer reproducibility were $1.1 \%$ and $2.2 \%$, respectively [18]. baPWV was defined as the mean of left and right baPWV. Systolic and diastolic blood pressures in the supine position, determined simultaneously with baPWV, were also expressed as the mean of left and right blood pressures.

\section{Statistical analyses}

All data are shown as means \pm SD. For statistical analyses, unpaired Student's $t$ test was used to compare between two groups, as appropriate. The relationship between FPG levels and different variables was assessed by linear univariate regression analyses. The StatView computer program (Version 5.0 for Windows; 

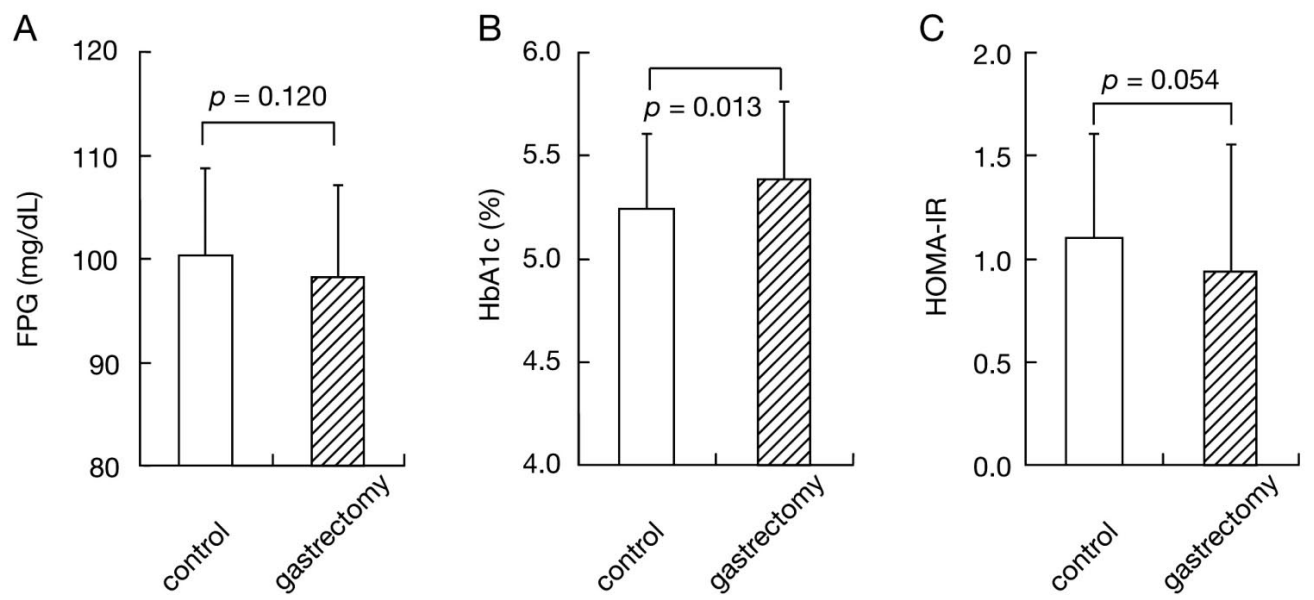

Fig. 1 Fasting plasma glucose (FPG) (A), HbA1c (B), and HOMA-IR (C) in 44 gastrectomized men and in 278 control men.
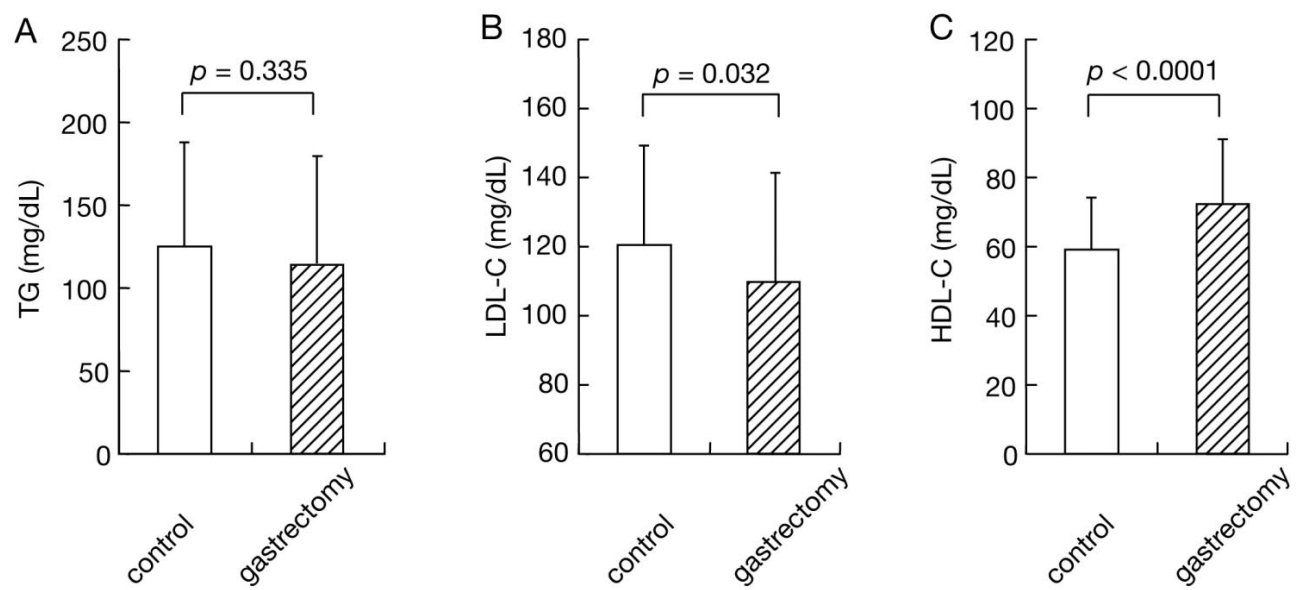

Fig. 2 Serum triglycerides (A), LDL-cholesterol (LDL-C) (B), and HDL-cholesterol (HDL-C) (C) in 44 gastrectomized men and in 278 control men.

Abacus Concepts, Berkeley, CA) was used for all statistical analyses. A $p$ value of $<0.05$ was considered to be statistically significant.

\section{Results}

FPG levels (98 $\pm 9 \mathrm{mg} / \mathrm{dL}$ vs. $100 \pm 8 \mathrm{mg} / \mathrm{dL}$, $p=0.120)$, as well as HOMA-IR $(0.93 \pm 0.62$ vs. 1.10 $\pm 0.51, p=0.275$; Fig. 1), were not different between groups, whereas $\mathrm{HbAlc}$ levels were significantly higher in the gastrectomized men ( $5.8 \pm 0.4 \%$ vs. 5.6 $\pm 0.4 \%, p=0.013$ ). Serum LDL-C concentrations were significantly lower in the gastrectomized men than in the control men $(110 \pm 31 \mathrm{mg} / \mathrm{dL}$ vs. $120 \pm 29 \mathrm{mg} / \mathrm{dL}$, $p=0.032$ ), while HDL-C concentrations were higher in the gastrectomized men ( $73 \pm 19 \mathrm{mg} / \mathrm{dL} v \mathrm{~s} .59 \pm 15 \mathrm{mg} /$ $\mathrm{dL}, p<0.0001$ ), and serum triglycerides levels were not different between groups $(115 \pm 65 \mathrm{mg} / \mathrm{dL} v \mathrm{~s} .125 \pm 63$ $\mathrm{mg} / \mathrm{dL}, p=0.335$; Fig. 2). Systolic and diastolic blood pressures in the gastrectomized men $(128 \pm 65 \mathrm{mmHg}$ and $81 \pm 14 \mathrm{mmHg}$ ) were significantly higher than those in the control men $(120 \pm 15 \mathrm{mmHg}, p=0.002$ and $75 \pm$ $11 \mathrm{mmHg}, p=0.002)$ (Fig. 3). Systolic $(130 \pm 19 \mathrm{mmHg}$ vs. $123 \pm 15 \mathrm{mmHg}, p=0.009)$ and diastolic blood pressures ( $82 \pm 13 \mathrm{mmHg}$ vs. $77 \pm 10 \mathrm{mmHg}, p=0.002$ ) in the supine position, as determined simultaneously with baPWV measurements, were also higher in the gastrectomized men compared to controls. However, resting heart rates were not different between groups (64.5 \pm $9.4 / \mathrm{min} v s$. $63.5 \pm 8.3 / \mathrm{min}, p=0.473$ ). Serum uric acid 

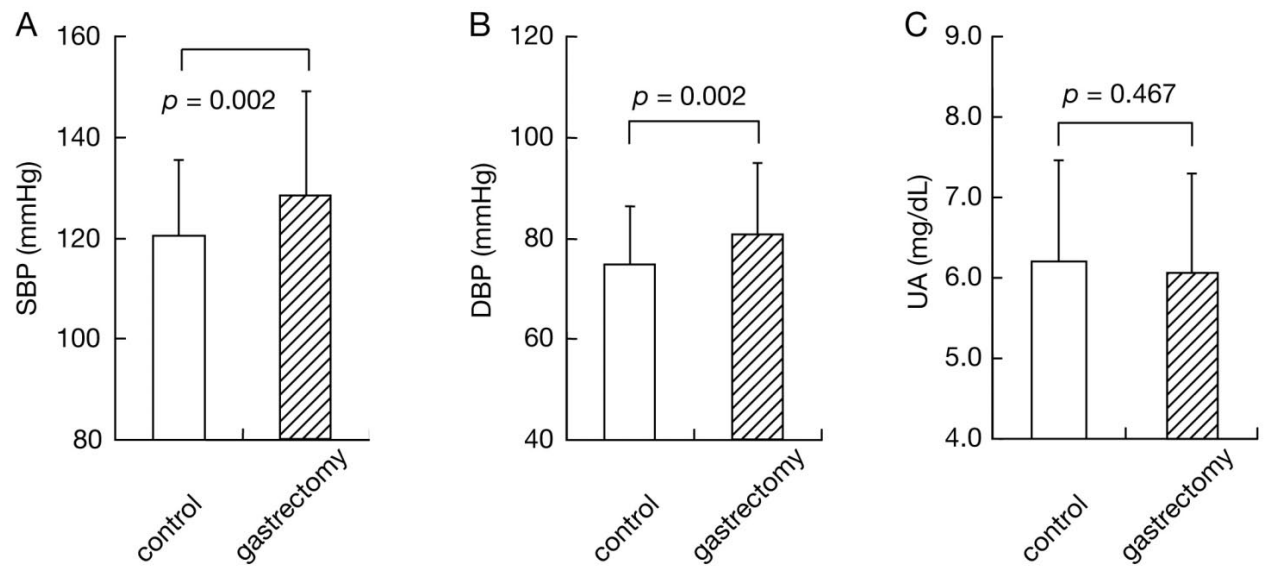

Fig. 3 Systolic blood pressure (SBP) (A), diastolic blood pressure (DBP) (B), and serum uric acid (UA) (C) in 44 gastrectomized men and in 278 control men.
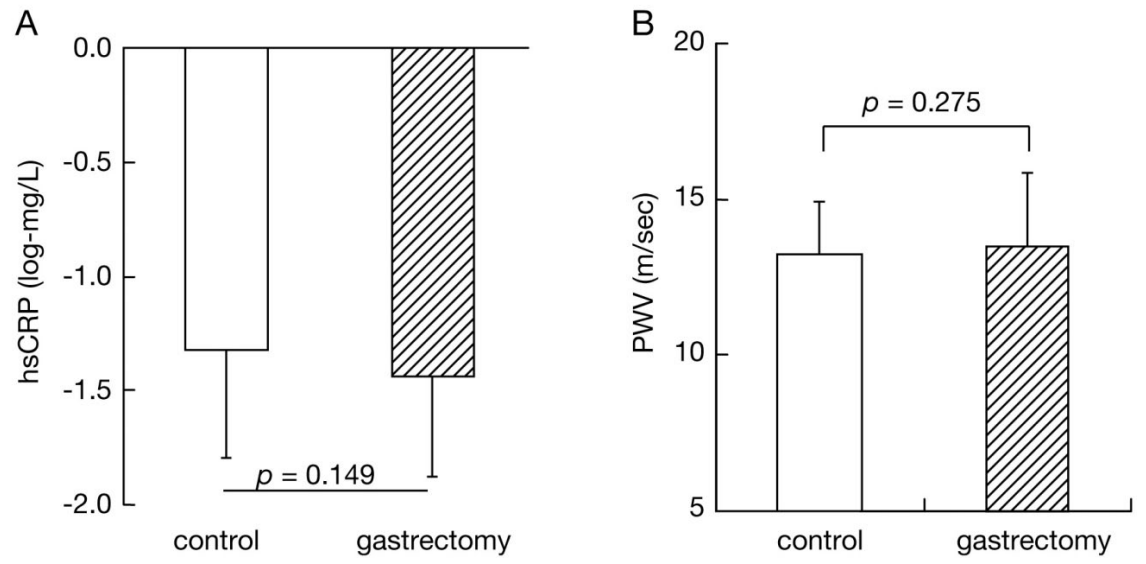

Fig. 4 High sensitivity C-reactive protein (hsCRP) (A) and brachial-ankle pulse wave velocity (baPWV) (B) in 44 gastrectomized men and in 278 control men.

(6.1 $\pm 1.2 \mathrm{mg} / \mathrm{dL} v s .6 .2 \pm 1.3 \mathrm{mg} / \mathrm{dL}, p=0.467)$, hsCRP (log-transformed-mg/L; $-1.43 \pm 0.45$ vs. $-1.32 \pm 0.47$, $p=0.149)$ and baPWV $(13.5 \pm 2.3 \mathrm{~m} / \mathrm{sec} v s .13 .2 \pm 1.7$ $\mathrm{m} / \mathrm{sec}, p=0.275$ ) were not different between gastrectomized versus control men (Fig. 4).

\section{Discussion}

In the present investigation, we examined the traditional atherosclerotic risk factors and surrogate markers for atherosclerosis in gastrectomized subjects. The rationale for this study was based on the observation that postprandial hyperglycemia, a known risk factor for atherosclerotic vascular diseases, is frequently observed in gastrectomized subjects. It has been shown that blood glucose and serum uric acid levels are lower, and that serum LDL-C and HDL-C levels are higher, in women versus men. In addition, it is well known that the incidence of cardiovascular disease, and the levels of hsCRP and baPWV, are lower in women than in men. Thus, to avoid the potential confounding effects of gender on the parameters studied, the present study focused solely on men. Furthermore, the control subjects in the present study comprised of age- and BMImatched men without a history of gastrectomy, as age and BMI both directly and indirectly influence atherosclerotic risk. It is generally accepted that the BMI of gastrectomized subjects is lower than that of non-gastrectomized subjects [3]. Recently, bariatric surgery, in addition to by-pass surgery, is offered as a treatment of marked obesity [20]. Therefore, in order to properly assess the results of the present study, the reduced BMI in response to gastrectomy must be considered.

Although FPG levels were not different between 
groups, HbAlc levels were significantly higher in the gastrectomized men. These results are consistent with a previous report demonstrating higher glycated albumin levels in gastrectomized subjects compared with control subjects [3]. Taken together, the gastrectomized men in the present study exhibited postprandial hyperglycemia. Compared to diabetic patients, gastrectomized patients experienced a shorter duration of postprandial hyperglycemia. However, a transient rise of plasma glucose levels can cause vascular endothelial injury [21], leading to the progression of atherosclerosis. Insulin resistance, as measured by HOMA-IR, was not significantly different between groups, but was numerically lower in the gastrectomized men. Alterations of hormonal milieu after gastrectomy may influence insulin sensitivity. Further studies including a larger subject sample are necessary to clarify this issue.

In the present study, gastrectomized men exhibited lower LDL-C and higher HDL-C in comparison to healthy controls, in a manner consistent with a previous report [9]. On conversely, we failed to show significant differences in serum triglycerides levels between groups, which is inconsistent with a previous finding of lower serum triglycerides levels in gastrectomized versus non-gastrectomized subjects [8]. The discrepancy between these studies may be due to the fact that the control population was BMI-matched in the current, but not in the previous study [8]. Thus, gastrectomy itself may be involved in the decreased LDL-C and the increased HDL-C levels, while decreased BMI related to gastrectomy results in decreased triglycerides levels.

Our study found that both systolic and diastolic blood pressures were higher among gastrectomized men. In contrast, the Honolulu Heart Study on JapaneseAmerican men reported that blood pressure in gastrectomized subjects was lower compared with the control population [8]. Again, such discrepant results may be caused by the BMI-matching of our control group. It has recently been shown that ghrelin, a peptide produced by the stomach, plays a role in reducing blood pressure [22], and low plasma ghrelin levels are associated with hypertension [23]. Since circulating ghrelin concentrations are depressed after gastrectomy [24], this may be the mechanism by which blood pressure was increased among the gastrectomized men in the present study.

The surrogate marker for atherosclerosis, baPWV, was not different between the two groups. To our knowledge, no prior report examined baPWV in gastrectomized subjects. However, baPWV is well known to be influenced by blood pressure [25]. Nevertheless, although systolic and diastolic blood pressures were significantly higher in the gastrectomized men than in the control men, baPWV was similar across the groups. Atherosclerosis progression is thought to be influenced by a balance between pro- and anti-atherogenic factors [26, 27]. Thus, in gastrectomized men, baPWV might not be elevated because of the balance between postprandial hyperglycemia, and increased blood pressures (pro-atherogenic), as well as decreased LDL-C and increased HDL-C (anti-atherogenic). In the future, other markers of atherosclerosis should be evaluated.

Gastrectomy alters secretions of various hormones, which may also influence atherosclerosis progression. For example, it has been shown that circulating glucagon-like peptide-1 (GLP-1) concentrations are increased in gastrectomized subjects [28]. Given that GLP-1 exerts an anti-atherogenic effect [29], a mechanism by which the increased GLP-1 in gastrectomized subjects prevents atherosclerosis progression may exist. Further studies will be needed to address these issues.

\section{Conflicts of Interest}

The authors declare no conflict of interest relevant to this manuscript.

\section{References}

1. Lawrence RD. (1974) Symptomless glycosuria: differentiation by sugar tolerance test. Med Clin North Am 31: 289-297.

2. Ceriello A. (1999) Hyperglycaemia: the bridge between non-enzymatic glycation and oxidative stress in the pathogenesis of diabetic complications. Diabetes Nutr Metab 12: 42-46.
3. Koga M, Murai J, Saito H, Mukai M, Matsumoto S, Kasayama S. (2010) Glycated albumin levels are higher relative to glycated haemoglobin levels in gastrectomized subjects. Ann Clin Biochem 47: 39-43.

4. The DECODE study group. (1999) Glucose tolerance and mortality: comparison of WHO and American Diabetes Association diagnostic criteria. Lancet 354: 
617-621.

5. Tominaga M, Eguchi H, Manaka H, Igarashi K, Kato T, Sekikawa A. (1999) Impaired glucose tolerance is a risk factor for cardiovascular disease, but not impaired fasting glucose. The Funagata Diabetes Study. Diabetes Care 22: 920-924.

6. Lundegårdh G, Helmick C, Zack M, Adami HO. (1994) Mortality among patients with partial gastrectomy for benign ulcer disease. Dig Dis Sci 39: 340-346.

7. von Holstein CS. (2000) Long-term prognosis after partial gastrectomy for gastroduodenal ulcer. World J Surg 24: 307-314.

8. Glober GA, Rhoads GG, Liu F, Kagan A. (1974) Long term results of gastrectomy with respect to blood lipids, blood pressure, weight and living habits. Ann Surg 179: 896-901.

9. Glober GA, Rhoads GG, Liu F, Kagan A. (1985) The effect of partial gastrectomy on lipoproteins and other characteristics. J Chronic Dis 38: 609-615.

10. Libby P, Ridker PM. (2004) Inflammation and atherosclerosis: role of C-reactive protein in risk assessment. Am J Med 116 Suppl 6A: 9S-16S.

11. Nichols WW. (2005) Clinical measurement of arterial stiffness obtained from noninvasive pressure wave forms. Am J Hypertens 18: 3S-10S.

12. Vogel RA, Benitez RM. (2000) Noninvasive assessment of cardiovascular risk: from Framingham to the future. Rev Cardiovasc Med 1: 34-42.

13. Schnedl WJ, Lahousen T, Wallner SJ, Krause R, Lipp RW. (2005) Silent hemoglobin variants and determination of $\mathrm{HbA}_{1 \mathrm{C}}$ with the high-resolution program of the HPLC HA-8160 hemoglobin analyzer. Clin Biochem 38: 88-91.

14. The Committee of Japan Diabetes Society on the diagnostic criteria of diabetes mellitus. (2010) Report of the Committee on the classification and diagnostic criteria of diabetes mellitus. J Diabetes Invest 1: 212-228.

15. Friedewald WT, Levy RI, Fredrickson DS. (1972) Estimation of the concentration of low-density lipoprotein cholesterol in plasma, without use of the preparative ultracentrifuge. Clin Chem 18: 499-502.

16. Hashimoto K, Kasayama S, Yamamoto H, Kurebayashi S, Kawase I, Koga M. (2004) Strong association of C-reactive protein with body mass index and 2-h postchallenge glucose in non-diabetic, non-smoker subjects without hypertension. Diabet Med 21: 581-585.

17. Matthews DR, Hosker JP, Rudenski AS, Naylor BA, Treacher DF, Turner RC. (1985) Homeostasis model assessment: insulin resistance and beta-cell function from fasting plasma glucose and insulin concentrations in man. Diabetologia 28: 412-419.

18. Kasayama S, Saito H, Mukai M, Koga M. (2005)
Insulin sensitivity independently influences brachial-ankle pulse wave velocity in non-diabetic subjects. Diabet Med 22: 1701-1706.

19. Kubo T, Miyata M, Minagoe S, Setoyama S, Maruyama I, Tei C. (2002) A simple oscillometric technique for determining new indices of arterial distensibility. Hypertens Res 25: 351-358.

20. Sjöström L, Narbro K, Sjöström CD, Karason K, Larsson B, Wedel H, Lystig T, Sullivan M, Bouchard C, Carlsson B, Bengtsson C, Dahlgren S, Gummesson A, Jacobson P, Karlsson J, Lindroos AK, Lönroth H, Näslund I, Olbers T, Stenlöf K, Torgerson J, Agren G, Carlsson LM, Swedish Obese Subjects Study. (2007) Effects of bariatric surgery on mortality in Swedish obese subjects. $N$ Engl J Med 357:741-752.

21. Otsuka A, Azuma K, Iesaki T, Sato F, Hirose T, Shimizu T, Tanaka Y, Daida H, Kawamori R, Watada H. (2005) Temporary hyperglycaemia provokes monocyte adhesion to endothelial cells in rat thoracic aorta. Diabetologia 48: 2667-2674.

22. Vlasova MA, Järvinen K, Herzig KH. (2009) Cardiovascular effects of ghrelin antagonist in conscious rats. Regul Pept 156: 72-76.

23. Pöykkö SM, Kellokoski E, Hörkkö S, Kauma H, Kesäniemi YA, Ukkola O.(2003) Low plasma ghrelin is associated with insulin resistance, hypertension, and the prevalence of type 2 diabetes. Diabetes 52: 2546-2553.

24. Santoro S, Milleo FQ, Malzoni CE, Klajner S, Borges PC, Santo MA, Campos FG, Artoni RF. (2008) Enterohormonal changes after digestive adaptation: five-year results of a surgical proposal to treat obesity and associated diseases. Obes Surg 18: 17-26.

25. Shirai K, Utino J, Otsuka K, Takata M. (2006) A novel blood pressure-independent arterial wall stiffness parameter; cardio-ankle vascular index (CAVI). J Atheroscler Thromb 13: 101-107.

26. Cullen P, Assmann G. (1999) High risk strategies for atherosclerosis. Clin Chim Acta 286: 31-45.

27. Kubo M, Kiyohara Y, Kato I, Tanizaki Y, Arima H, Tanaka K, Nakamura H, Okubo K, Iida M. (2003) Trends in the incidence, mortality, and survival rate of cardiovascular disease in a Japanese community: the Hisayama study. Stroke 34: 2349-2354.

28. Miholic J, Orskov C, Holst JJ, Kotzerke J, Meyer HJ. (1991) Emptying of the gastric substitute, glucagon-like peptide-1 (GLP-1), and reactive hypoglycemia after total gastrectomy. Dig Dis Sci 36: 1361-1370.

29. Rizzo M, Rizvi AA, Spinas GA, Rini GB, Berneis K. (2009) Glucose lowering and anti-atherogenic effects of incretin-based therapies: GLP-1 analogues and DPP-4inhibitors. Expert Opin Investig Drugs 18: 1495-1503. 